Research Article

\title{
Impact of Immunization Strategies on the Dynamics of Social Contagions
}

\author{
Hao Peng, ${ }^{1,2}$ Wangxin Peng, ${ }^{1}$ Dandan Zhao $\mathbb{D}^{1},{ }^{1}$ Zhaolong Hu, ${ }^{1}$ Jianmin Han, ${ }^{1}$ \\ and Zhonglong Zheng ${ }^{1}$ \\ ${ }^{1}$ College of Mathematics and Computer Science, Zhejiang Normal University, Jinhua 321004, Zhejiang, China \\ ${ }^{2}$ Shanghai Key Laboratory of Integrated Administration Technologies for Information Security, Shanghai 200240, China \\ Correspondence should be addressed to Dandan Zhao; ddzhao@zjnu.edu.cn
}

Received 29 May 2020; Accepted 22 June 2020; Published 1 August 2020

Guest Editor: Wei Wang

Copyright (C) 2020 Hao Peng et al. This is an open access article distributed under the Creative Commons Attribution License, which permits unrestricted use, distribution, and reproduction in any medium, provided the original work is properly cited.

\begin{abstract}
Immunization strategies on complex networks are effective methods to control the spreading dynamics on complex networks, which change the topology and connectivity of the underlying network, thereby affecting the dynamics process of propagation. Here, we use a non-Markovian threshold model to study the impact of immunization strategies on social contagions, in which the immune index greater than (or equal to) 0 corresponds to targeted (random) immunization, and when the immune index is less than 0 , the probability of an individual being immunized is inversely related to the degree of the individual. A generalized edgebased compartmental theory is developed to analyze the dynamics of social contagions under immunization, and theoretical predictions are very consistent with simulation results. We find that increasing the immune index or increasing the immune ratio will reduce the final adoption size and increase the outbreak threshold, in other words, make the residual network after immunization not conducive to social contagions. Interestingly, enhancing the network heterogeneity is proved to help improve the immune efficiency of targeted immunization. Besides, the dependence of the outbreak threshold on the network heterogeneity is correlated with the immune ratio and immune index.
\end{abstract}

\section{Introduction}

In many fields, such as sociology and biology, the diffusion mechanisms of some real spreading processes can be essentially described as the spreading dynamics on complex networks [1-6]. Unlike biological contagions, social contagions, such as rumor diffusion [7], behavior spreading $[8,9]$, information diffusion [10], the adoption of new product [11], the diffusion of microfinance [12], and innovation diffusion $[13,14]$, have a social reinforcement effect. The credibility of behavior or information usually needs to be confirmed multiple times to reduce risks. That is, the adoption probability of a certain contact depends on the amount of previous contacts. More specifically, for susceptible individuals, before the number of exposures to the same information reaches the adoption threshold, the more times the same information is received from different neighbors, the greater the adoption possibility [15]. In the linear threshold model $[16,17]$, the dynamics of social contagions was regarded as a Markov process. In recent years, researchers found that the social reinforcement effect is based on the memory characteristic, and many distinct non-Markovian models [18-28] have been proposed. Wang et al. [18] proposed an edge-based compartmental (EBC) theory to qualitatively analyze the steady state of the dynamics of social contagions with nonredundant information memory characteristic and found that the change of some structural parameters (e.g., the heterogeneity of the network structure) or dynamic factors (e.g., the adoption threshold) will cause the crossover phenomenon of the system. Backlund et al. [19] found that sufficiently long time windows promote the global cascade of adoption on temporal networks. About the effects of community structure on social contagions on temporal networks, Liu et al. [20] found 
that when the information transmission rate takes some appropriate values, increasing the community strength can promote the spreading of behavior in communities, and the optimal community strength can maximize the final adoption size. Besides, the heterogeneity of link weights [21], heterogeneous adoption threshold [22-24], and time delays [25] also affect the dynamics of social contagions.

In addition to proposing some more realistic propagation models, taking appropriate immunization strategies is also an effective way to forecast and control the spreading dynamics on complex networks. In the early years, researchers proposed three classic immunization strategies on complex networks, namely, random immunization [29], targeted immunization [29], and acquaintance immunization $[30,31]$. From efficiency, acquaintance immunization is more efficient than random immunization and is slightly less efficient than targeted immunization. In another respect, targeted immunization needs to know the degree of each individual in the network, which is very difficult for a dynamically developing real network. However, acquaintance immunization is based on the local information of the network and does not need to identify hub individuals. Based on the three typical immunization strategies, some improving immunization strategies were proposed [32-35]. Considering that the underlying network plays an important role in the spreading dynamics on complex networks, some immunization strategies that take the role of the multiplex network [36, 37], community structure [38, 39], weighted network [40,41] or time-varying network [42] into account have been proposed. The existing studies have found that immunizing important nodes in the network can effectively suppress or control the epidemic spreading.

However, there is still a lack of systematic theoretical and numerical simulations to address how immunization strategies affect the dynamics of social contagions. For this purpose, we use the non-Markovian susceptible-adoptedrecovered (SAR) threshold model to study the effects of different immunization strategies on social contagions, in which we select different types of immunization strategies by adjusting the value of the immune index $\alpha$. Then, we develop a generalized EBC theory to analyze the dynamics of social contagions under immunization. Through theoretical predictions and numerical simulations on scale-free (SF) networks, we found that (i) increasing the immune index will reduce the final adoption size and increase the outbreak threshold (threshold for short) when the immune ratio is the same; (ii) increasing the immune ratio will decrease the final adoption size and increase the threshold; (iii) when the immune ratio is small, the threshold decreases as the network heterogeneity increases (as $\gamma_{D}$ decreases), but when the immune ratio is relatively large and under targeted immunization, the threshold increases as the network heterogeneity increases.

The remainder of the paper is organized as follows. In Section 2, we describe the dynamics of social contagions. Section 3 gives theoretical analysis through the EBC theory. Section 4 presents numerical simulations. Finally, the conclusions are presented in section 5 .

\section{Model Description}

The non-Markovian SAR threshold model is a general model to describe the dynamics of social contagions with nonredundant information memory characteristic; thus, we adopt it to describe the dynamics of social contagions under immunization and study the effects of different immunization strategies on the final adoption size and outbreak threshold of social contagions. There are the following three states of the individual: susceptible state $(S)$, adopted state $(A)$, and recovered state $(R)$. Susceptible individuals will not adopt the behavior unless the amount of nonredundant information received from neighbors is not less than the adoption threshold. Adopted individuals have adopted the behavior and can transmit the behavioral information to their susceptible neighbors with probability $\lambda$. If the adopted individual successfully recovers as the recovered individual with probability $\gamma$, then, in the subsequent social contagion process, the recovered individual will not share information with his/her neighbors and will always remain in the recovered state. Before the social contagion terminates, the state of individuals will change dynamically.

The immunization strategies in social networks refer to selecting some individuals in the network to be immune to social contagion. That is to say, these selected individuals and their links will be removed from the initial network; thus, they cannot participate in social contagion, which plays a significant role in the prevention and control of social contagions. In real-world networks, the probability of an individual being immune to social contagion is correlated with the number of his/her links (the degree), and each individual $i$ with $k_{i}$ links is assigned a value $M_{\alpha}\left(k_{i}\right)$, which denotes the probability of individual $i$ being immunized. According to the family of functions [43-45], the expression of $M_{\alpha}\left(k_{i}\right)$ is

$$
M_{\alpha}\left(k_{i}\right)=\frac{k_{i}^{\alpha}}{\sum_{i=1}^{N} k_{i}^{\alpha}}, \quad-\infty<\alpha<+\infty,
$$

where the value of immune index $\alpha$ determines the correlation between an individual's degree and the probability of the individual being immune to social contagion. The case $\alpha>0$ represents targeted immunization (TI), which means that individuals with higher degrees are more likely to be immunized. Case $\alpha=1$, especially, corresponds to acquaintance immunization (AI), which is a localized immunization; that is, random neighbors of random individuals are chosen to be immunized. For $\alpha=0$, all individuals have the same probability of being immunized (i.e., $\left.M_{0}\left(k_{i}\right)=1 / N\right)$, which represents random immunization (RI). Note that when $\alpha<0$, individuals with lower degrees have a higher probability to be immunized, which is the opposite of when $\alpha>0$.

In order to facilitate the analysis, the adoption threshold of all individuals is set to $T$. In the initial stage, a fraction $1-q$ individuals are selected to be immunized first, which causes the topology of the underlying network to change. In other words, the residual network consists of the remaining $q$ proportion individuals and their links, and these 
individuals can participate in the subsequent social contagion. Specifically, we call the immunized individuals as invalid individuals, and the remaining unimmunized individuals are called valid individuals. Then, a proportion $\rho_{0}$ of valid individuals are selected randomly as the adopted individuals for triggering social contagion, and the remaining valid individuals are susceptible individuals and have not obtained any behavioral information. At each time step, in the residual network, each adopted individual $j$ can transmit the behavioral information to its susceptible neighbors with probability $\lambda$ in a nonredundant transmission way (i.e., a piece of information can only be passed once through an edge). Among valid individuals, if a random susceptible individual $i$ receives the information from individual $j$, the amount $m_{i}$ of nonredundant information received by individual $i$ will increase by one. When $m_{i} \geq T$, individual $i$ adopts the behavior; otherwise it is still in the susceptible state. Moreover, individuals in the adopted state try to recover with probability $\gamma$ at each time step. If there are no adopted individuals in the residual network after immunization, the social contagion ends.

\section{Theoretical Analysis}

In this section, based on the non-Markovian SAR threshold model, a general EBC theory is developed to analyze the dynamics of social contagion under immunization. The three variables $S(t), A(t)$, and $R(t)$, respectively, represent the proportion of individuals in the susceptible, adopted, and recovered states at time step $t$. If an immunization measure is implemented before social contagion occurs (i.e., the immune ratio $1-q \neq 0$ ), these immunized individuals will be removed from the network; thus, $S(t)+A(t)+R(t)=q<1$. When $t \longrightarrow \infty$, the dynamic process of social contagion reaches the final state (i.e., the state of all individuals no longer changes), and $R(\infty)$ represents the percentage of individuals who have adopted the behavior (the final adoption size for short).

After the initial immunization, these immunized individuals and their links are removed from the initial network, which results in a change in the degree of the neighbors of these immunized individuals. Therefore, we first need to calculate the degree distribution $P_{q}(k)$ of the residual network after immunization, where $q$ is the ratio of valid (unimmunized) individuals. According to the method in $[45,46]$, we let $G_{q}(k)$ denote the amount of valid individuals with degree $k$, and the degree distribution $P_{q}(k)$ of valid individuals is given as

$$
P_{q}(k)=\frac{G_{q}(k)}{q N} .
$$

When another individual is immunized and removed from the network, by using (1), the expression of $P_{q}(k)$ changes as

$$
G_{(q-1 / N)}(k)=G_{q}(k)-\frac{P_{q}(k) k^{\alpha}}{\left\langle k^{\alpha}(q)\right\rangle}
$$

where $\left\langle k^{\alpha}(q)\right\rangle=\sum_{k} P_{q}(k) k^{\alpha}$. In the limit of $N \longrightarrow \infty$, (3) can be written as the derivative equation of $G_{q}(k)$,

$$
\frac{\mathrm{d} G_{q}(k)}{\mathrm{d} q}=N \frac{P_{q}(k) k^{\alpha}}{\left\langle k^{\alpha}(q)\right\rangle} \text {. }
$$

Taking the derivative of (2) with respect to $q$ and combining (4), we have

$$
-q \frac{\mathrm{d} P_{q}(k)}{\mathrm{d} q}=P_{q}(k)-\frac{P_{q}(k) k^{\alpha}}{\left\langle k^{\alpha}(q)\right\rangle} .
$$

By direct differentiation and defining the function $F_{\alpha}(\psi)=\sum_{k} p(k) \psi^{k^{\alpha}}$ and variable $\psi=F_{\alpha}^{-1}(q)$ [46], we can get the expressions of $P_{q}(k)$ and $\left\langle k^{\alpha}(q)\right\rangle$ as

$$
\begin{aligned}
P_{q}(k) & =\frac{1}{q} p(k) \psi^{k^{\alpha}}, \\
\left\langle k^{\alpha}(q)\right\rangle & =\frac{\psi F_{\alpha}^{\prime}(\psi)}{F_{\alpha}(\psi)},
\end{aligned}
$$

respectively.

After the immunization strategy is implemented, for a random individual $i$ with degree $k$, the probability that it receives $m$ pieces of nonredundant behavioral information by time $t$ is

$$
\pi(k, m, \tau(t))=\left(1-\rho_{0}\right) C_{k}^{m} \tau(t)^{k-m}[1-\tau(t)]^{m},
$$

where $1-\rho_{0}$ is the initial fraction of susceptible individuals after immunization and $\tau(t)$ represents the probability that by time $t$, a random edge of individual $j$ has not transmitted the information to a susceptible neighbor $i$. According to the condition of a susceptible individual adopting behavior (i.e., $m \geq T$ ), the probability that individual $i$ remains susceptible at time $t$ is

$$
s(k, t)=\sum_{m=0}^{T-1} \pi(k, m, \tau(t)) .
$$

Considering all possible values of the degree $k$ of valid individuals, the fraction of susceptible individuals in the residual network at time $t$ is

$$
S(t)=\sum_{k} P_{q}(k) s(k, t) .
$$

It is worth noting that the neighbor of individual $i$ may be in one of three states; therefore the expression of $\tau(t)$ is defined as

$$
\tau(t)=\chi_{S}(t)+\chi_{A}(t)+\chi_{R}(t),
$$

where $\chi_{S}(t), \chi_{A}(t)$, and $\chi_{R}(t)$, respectively, represent the probabilities that a neighbor of individual $i$ is in the susceptible, adopted, or recovered states and has not transmitted the information to its neighbors by time $t$.

Next, the evolution expression of $\tau(t)$ will be solved. Individual $i$ is assumed to be in the cavity state [47, 48], i.e., it can only receive information from its neighbors but cannot transmit information to its neighbors. Therefore, the susceptible neighbor $j$ with degree $k^{\prime}$ of individual $i$ can only receive information from its $k^{\prime}-1$ neighbors except 
neighbor $i$. Similarly, the probability that individual $j$ acquires $m$ pieces of information by time $t$ is

$$
\mu\left(k^{\prime}, m, \tau(t)\right)=C_{k^{\prime}-1}^{m} \tau(t)^{k^{\prime}-m-1}[1-\tau(t)]^{m} .
$$

Consequently, the probability that individual $j$ remains in the susceptible state at time $t$ is $\psi\left(k^{\prime}, t\right)=$ $\sum_{m=0}^{T-1} \mu\left(k^{\prime}, m, \tau(t)\right)$. The probability that individual $i$ is connected to a neighbor with degree $k^{\prime}$ is $k^{\prime} P_{q}\left(k^{\prime}\right) /\langle k\rangle$; thus, we have

$$
\chi_{S}(t)=\frac{1-\rho_{0}}{\langle k\rangle} \sum_{k^{\prime}} k^{\prime} P_{q}\left(k^{\prime}\right) \psi\left(k^{\prime}, t\right)
$$

When the information fails to be transmitted through an edge with probability $1-\lambda$ and the connected adopted individual recovers with probability $\gamma, \chi_{R}(t)$ increases. Thus, the evolution of $\chi_{R}(t)$ is obtained as

$$
\frac{\mathrm{d} \chi_{R}(t)}{\mathrm{d} t}=\gamma(1-\lambda) \chi_{A}(t) .
$$

At time $t$, the adopted individuals transmit information to their susceptible neighbors with probability $\lambda$; thus, the evolution of $\tau(t)$ is

$$
\frac{\mathrm{d} \tau(t)}{\mathrm{d} t}=-\lambda \chi_{A}(t)
$$

Combining (14) and (15), we have $\chi_{R}(t)=\gamma(1-\lambda)$ $[1-\tau(t)] / \lambda$. Then, by substituting the expressions of $\chi_{R}(t)$ and $\chi_{S}(t)$ into (11), we get the expression of $\chi_{A}(t)$. Thus, (15) is rewritten as

$$
\begin{aligned}
\frac{\mathrm{d} \tau(t)}{\mathrm{d} t}= & -\lambda\left[\tau(t)-\frac{1-\rho_{0}}{\langle k\rangle} \sum_{k^{\prime}} k^{\prime} P_{q}\left(k^{\prime}\right) \psi\left(k^{\prime}, t\right)\right] \\
& +\gamma(1-\lambda)[1-\tau(t)] .
\end{aligned}
$$

As we know, susceptible individuals become adopted individuals once they adopt the behavior, and adopted individuals recover with probability $\gamma$; thus, the time evolution of $A(t)$ and $R(t)$ is

$$
\begin{aligned}
& \frac{\mathrm{d} A(t)}{\mathrm{d} t}=-\frac{\mathrm{d} S(t)}{\mathrm{d} t}-\gamma A(t), \\
& \frac{\mathrm{d} R(t)}{\mathrm{d} t}=\gamma A(t),
\end{aligned}
$$

respectively. By numerically integrating (10) and ((17)-(18)), the pattern of $R(t)$ versus $t$ is given. When the process of social contagion reaches a final state (i.e., $t \longrightarrow \infty), R(\infty)$ is the final adoption size.

\section{Numerical Simulations and Analysis}

In this section, we report the theoretical and numerical simulations on SF networks [49] with degree distribution $p(k) \sim k^{-\gamma_{D}}$, where $\gamma_{D}$ denotes degree exponent, and the structural heterogeneity of the network decreases as $\gamma_{D}$ increases. Unless otherwise specified, the network size, maximum degree, average degree, initial seeds ratio, adoption threshold, and recovery probability are set to be $N=10000, \quad k_{\max } \sim \sqrt{N},\langle k\rangle=10, \rho_{0}=0.1, T=3$, and $\gamma=1.0$, respectively. The outbreak threshold $\lambda_{c}$ indicates the critical value of information transmission probability. $\lambda_{c}$ can be determined by calculating the relative variance of $R(\infty)$ [50], which is defined as

$$
\Delta=\frac{\langle R(\infty)-\langle R(\infty)\rangle\rangle^{2}}{\langle R(\infty)\rangle^{2}}
$$

where $\langle\cdots\rangle$ denotes ensemble averaging, and $10^{3}$ independent simulations on a fixed network are performed to calculate the simulation value of the outbreak threshold. $\Delta$ reaches its peak at $\lambda_{c}$.

Figure 1 shows the dependence of the final adoption size $R(\infty)$ on the information transmission probability $\lambda$ under different immune ratio $1-q$, when the immune index $\alpha=3$, 0 and -3 , respectively. The lines from the theory are very consistent with these symbols representing the simulation results. When $\alpha=3$ (see Figure 1(a)), individuals with large degrees (hub individuals) in the network are given priority to be immunized, and a small immune ratio $1-q$ can have a great impact on the network. Therefore, as $1-q$ increases, $R(\infty)$ versus $\lambda$ pattern is first continuous and then becomes discontinuous; finally, the social contagion ends.

Notably, under targeted immunization, a very small immune ratio (e.g., $1-q=0.14$ ) can effectively suppress social contagion. When $\alpha=0$ (see Figure 1(b)), a number of individuals are randomly selected for immunization. In this case, with the increase of $1-q, R(\infty)$ first continuously increases with $\lambda$, and a large immune ratio (e.g., $1-q=0.6)$ can inhibit the outbreak of social contagion. When $\alpha=-3$ (see Figure 1(c)), individuals with small degrees are preferentially immunized. Immunizing some individuals with small degrees has less impact on network connectivity, so when the immune ratio is large (e.g., $1-q=0.8$ ), the connectivity of the residual network after immunization still supports the spread of information. By comparing Figures 1(a)-1(c), we note that targeted immunization has the strongest immune efficiency; that is, the increase of $\alpha$ will induce a better inhibitory effect on social contagions.

It can be seen from the above that the value of the immune index $\alpha$ has a significant impact on social contagions. We further investigate the effects of $\alpha$ on the final adoption size $R(\infty)$ and threshold $\lambda_{c}$ in Figure 2. As shown in Figure 2(a), $R(\infty)$ shows a downward trend as $\alpha$ increases, while in Figure 2(b), $\lambda_{c}$ increases as $\alpha$ increases, and $\lambda_{c}=1$ means that social contagion cannot occur. In Figure $2(a)$, the theoretical results (lines) agree well with the simulated results (symbols). This phenomenon can be understood as, with the increase of $\alpha$, a growing number of hub individuals are immunized, which is not conductive to social contagion.

More specifically, when the network heterogeneity is weak and a small number of individuals are immunized (i.e., $\gamma_{D}=4$ and $\left.1-q=0.05\right)$, the increase of $\alpha$ has little impact 


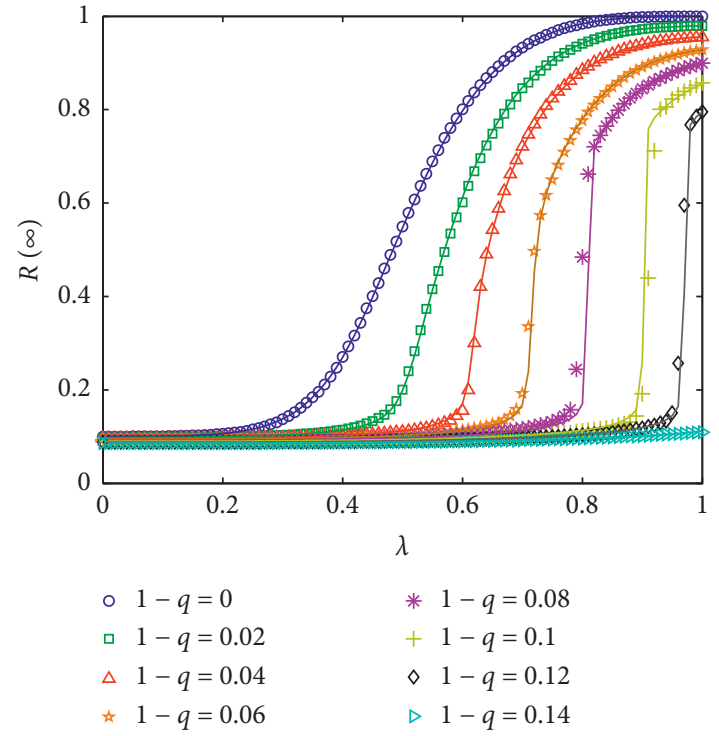

(a)

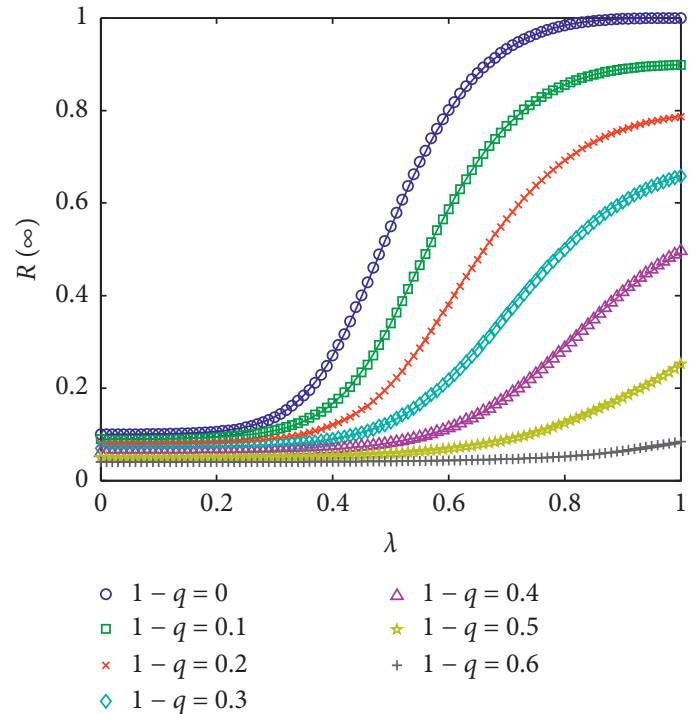

(b)

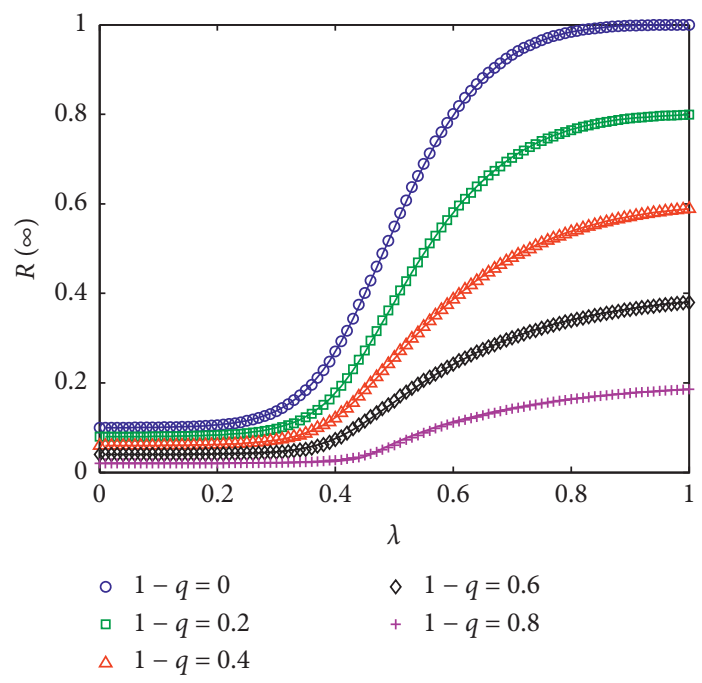

(c)

Figure 1: The final adoption size $R(\infty)$ versus the information transmission probability $\lambda$ on SF network with degree exponent $\gamma_{D}=3$ for (a) $\alpha=3$, (b) $\alpha=0$, and (c) $\alpha=-3$. Theoretical results (lines) agree well with simulated results (symbols).

on the network structure; thus, as $\alpha$ increases, $R(\infty)$ is basically unchanged and $\lambda_{c}$ increases slowly (see the green line in Figure 2). However, when the network heterogeneity is strong $\left(\gamma_{D}=2.2\right)$, the increase of $\alpha$ has a great impact on network connectivity, and especially when $\alpha>0$ and a relatively large number of individuals are immunized $(1-q=0.2), R(\infty)$ decreases sharply as $\alpha$ increases (see the red line in Figure 2(a)) and $\lambda_{c}$ increases rapidly as $\alpha$ increases (see the red line in Figure 2(b)). In addition, we find from Figure 2(a) that network heterogeneity and immune ratio play a significant role in the effect of immunization strategies. Specifically, for the same immune ratio, the targeted immunization on the strong heterogeneous network has a better immune effect than the targeted immunization on the weak heterogeneous network, and the larger the immune ratio, the better the immune effect.
To study the effects of the immune ratio $1-q$ on social contagion, we show the dependence of $R(\infty)$ and $\lambda_{c}$ on $1-q$ in Figure 3. In Figure 3(a), the lines from the theory are very consistent with these simulation results (symbols). Obviously, the increase of $1-q$ causes $R(\infty)$ to decrease (see Figure 3(a)) and causes $\lambda_{c}$ to increases (see Figure 3(b)). Since increasing $1-q$ means that a growing number of individuals are immunized, it will greatly disrupt the connectivity of the network. Specifically, when $\alpha=4$ (see the black and green lines in Figure 3), the hub individuals are immunized preferentially, as $1-q$ increases, $R(\infty)$ decreases sharply and $\lambda_{c}$ increases rapidly, and social contagion cannot break out when $1-q$ is approximately 0.2 . When $\alpha=0$ (see the blue and red lines in Figure 3), the individuals in the network are randomly immunized, the connectivity of the network is not seriously damaged, that is, there are still 


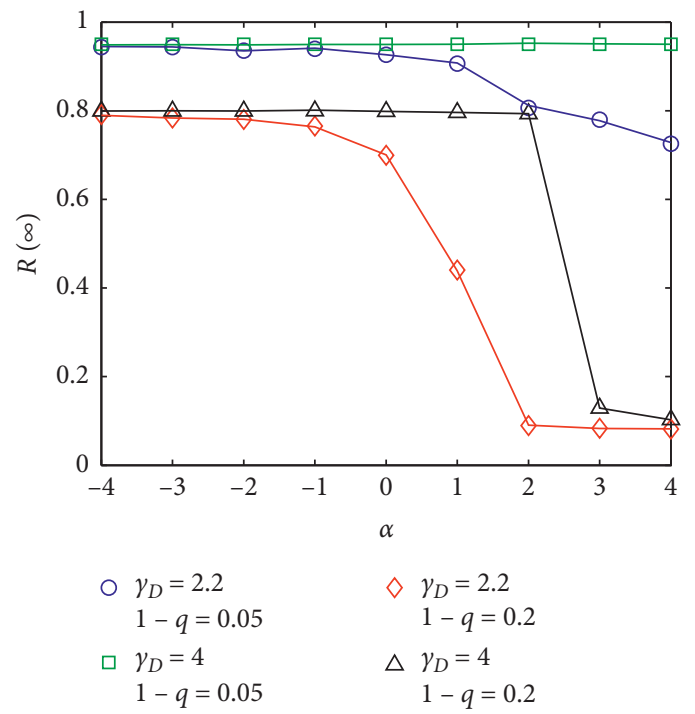

(a)

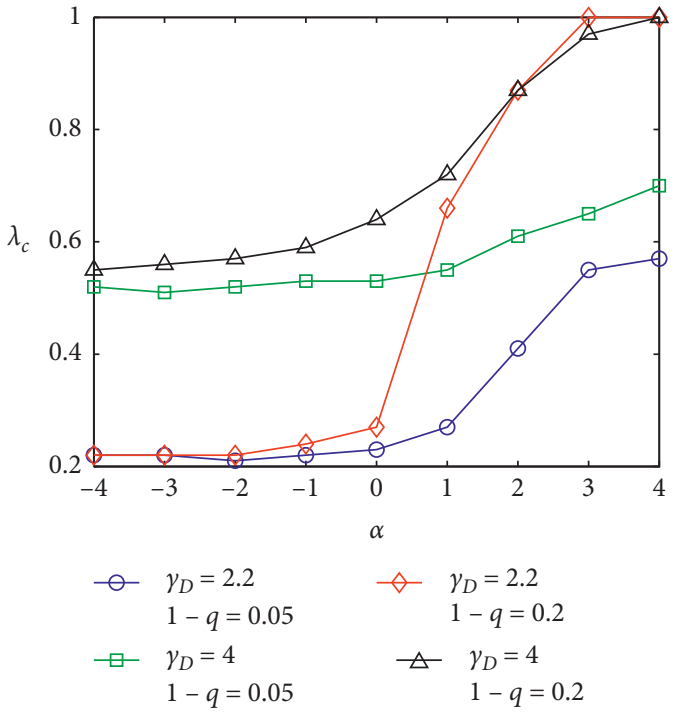

(b)

Figure 2: The effects of the immune index $\alpha$ on social contagions under different degree exponent $\gamma_{D}$ and immune ratio $1-q$ : (a) the final adoption size $R(\infty)$ versus $\alpha$ when $\lambda=1$ and (b) the threshold $\lambda_{c}$ versus $\alpha$.

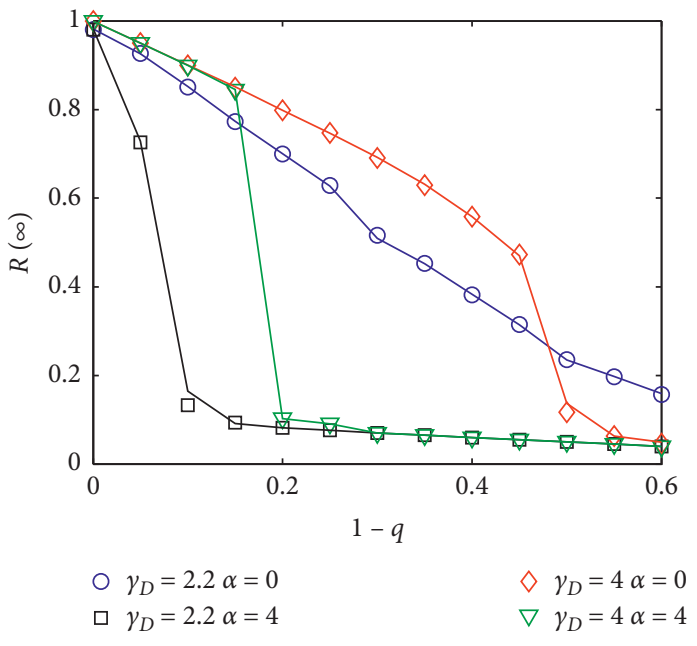

(a)

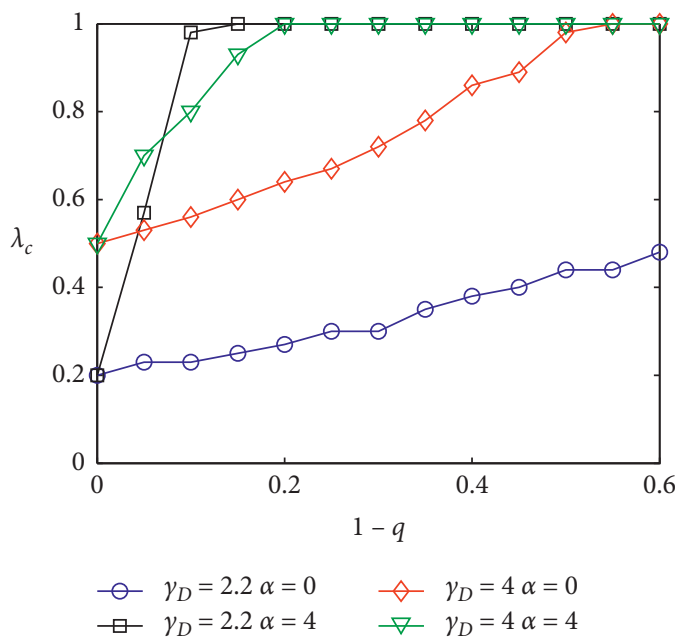

(b)

Figure 3: The effects of the immune ratio $1-q$ on social contagions under different degree exponent $\gamma_{D}$ and immune index $\alpha$, (a) the final adoption size $R(\infty)$ versus $1-q$ when $\lambda=1$ and (b) the threshold $\lambda_{c}$ versus $1-q$.

some individuals with large degrees in the residual network after random immunization; thus, the behavior can be spread on the residual network. In addition, the stronger the heterogeneity of the degree distribution, the more the hub individuals in the residual network after random immunization. As a result, when $\gamma_{D}=2.2$ and $\alpha=0$ (see the blue lines in Figure 3), the increase of $1-q$ has relatively minimal impact on the network structure; in other words, the system is more robust and stable.

The above studies have revealed that network heterogeneity has an important influence on the steady state of social contagion, for which the effects of the degree exponent $\gamma_{D}$ and the information transmission probability $\lambda$ on the final adoption size $R(\infty)$ are studied in Figure 4. For $1-q=0.05$, Figures $4(\mathrm{a})$ and $4(\mathrm{c})$ show the theoretical results of $R(\infty)$ in the plane $\lambda-\gamma_{D}$ for $\alpha=0$ and 4 , respectively. For $1-q=0.1$, Figures $4(\mathrm{e})$ and $4(\mathrm{~g})$ present the theoretical results of $R(\infty)$ for $\alpha=0$ and 4 , respectively. Figures 4(b), 4(d), 4(f), and 4(h) are the corresponding simulation results of Figures 4(a), 4(c), 4(e), and 4(g), respectively. The white circles in Figure 4 are simulated results of $\lambda_{c}$.

Overall, there is a remarkable agreement between theory and numerics in terms of the value of $R(\infty)$. For the case that $1-q$ is small (e.g., $1-q=0.05$ ), because only a small proportion of individuals are immunized, no matter whether 


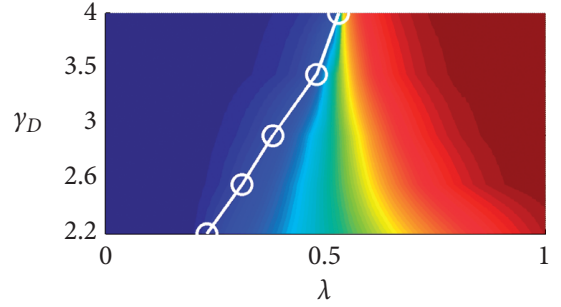

(a)

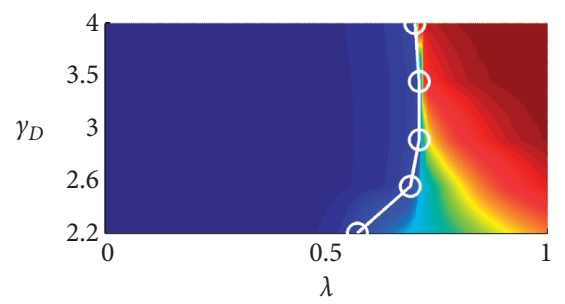

(c)

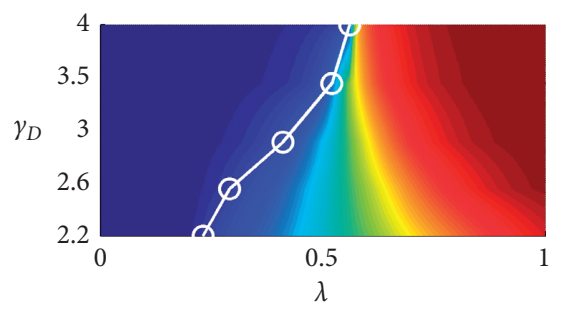

(e)

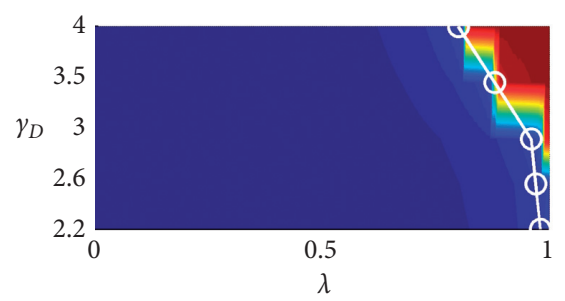

(g)

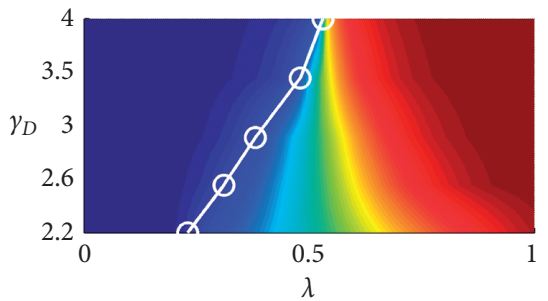

(b)

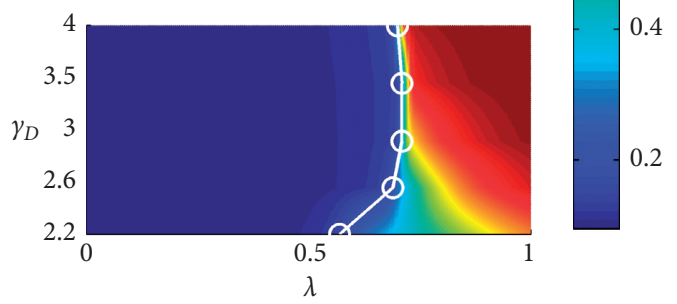

(d)

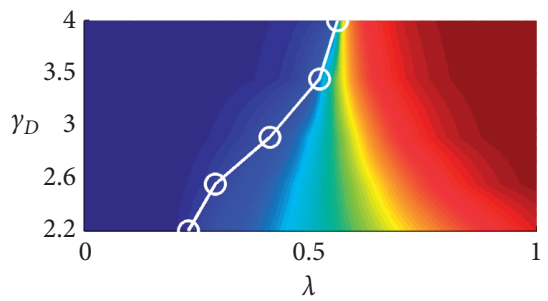

(f)

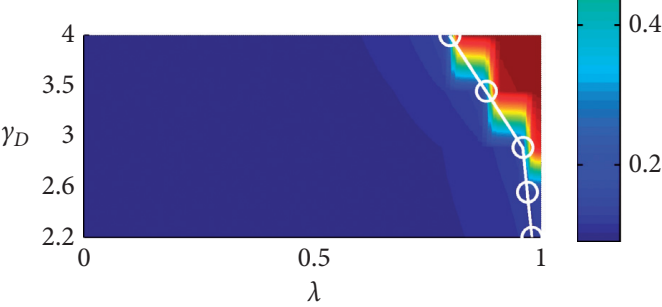

(h)

Figure 4: The final adoption size $R(\infty)$ versus information transmission probability $\lambda$ and degree exponent $\gamma_{D}$ on SF networks. The colorcoded values of $R(\infty)$ from theoretical solutions in the plane $\lambda-\gamma_{D}$ for (a) $\alpha=0,1-q=0.05$, (c) $\alpha=4,1-q=0.05$, (e) $\alpha=0,1-q=0.1$, and (g) $\alpha=4,1-q=0.1$. The corresponding simulated results for (b) $\alpha=0,1-q=0.05$, (d) $\alpha=4,1-q=0.05$, (f) $\alpha=0,1-q=0.1$, and (h) $\alpha=4,1-q=0.1$. The white circles are the simulated results of $\lambda_{c}$.

$\alpha=0$ (random immunization) or $\alpha=4$ (targeted immunization), there are still a large number of hub individuals in the remanent network after immunization; that is, the structure of the network has not been greatly affected. Hub individuals are conducive to social contagion, and the smaller $\gamma_{D}$, the more the hub individuals in the network, so $\lambda_{c}$ increases as $\gamma_{D}$ increases (see Figures 4(a) and 4(c)). Nevertheless, as $1-q$ increases to a relatively large value (e.g., $1-q=0.1$ ), the change trend of $\lambda_{c}$ depends on the value of $\alpha$. To be specific, when a random immunization strategy is adopted $(\alpha=0)$, after selecting some individuals for immunization with the same probability, there are still some hub individuals in the residual network, so $\lambda_{c}$ increases as $\gamma_{D}$ increases (see Figure 4(e)). Conversely, when a targeted immunization strategy is adopted $(\alpha=4), \lambda_{c}$ decreases as $\gamma_{D}$ increases (see Figure $4(\mathrm{~g})$ ). In this case, when $\gamma_{D}$ is relatively small, almost all hub individuals will be immunized, which will seriously change the topology structure of the network, so it is not conducive to the spread of behavior, and even behavior cannot be spread (i.e., $\lambda_{c}$ is approximately $1)$.

\section{Conclusions}

In summary, in this paper, we have systematically studied the effects of degree-based immunization strategies on the dynamics of social contagions. Using a non-Markovian SAR threshold model, we described the dynamics of social contagions under immunization, in which the adoption threshold of all individuals is the same, and the immune probability of each individual is related to its degree and the immune index. The type of immunization strategy depends on the size of the immune index. To give a dynamical analysis of social contagions under immunization, we developed a generalized edged-based compartmental theory. The predictions from theory are very consistent with these 
simulation results on SF networks. Firstly, we found that targeted immunization can produce the best immune effect on social contagions. In addition, we found that increasing the immune index or immune ratio will decrease the final adoption size and increase the threshold. Furthermore, we noted that when the immune ratio is small, increasing the network heterogeneity will decrease the threshold. Conversely, when targeted immunization is adopted and the immunization ratio is relatively large, the threshold increases as the network heterogeneity increases. This work just provides a starting point to understand the effects of immunization strategies on social contagions, which can provide some implications for managing and controlling social contagions. A more in-depth understanding of the role of immunization strategies in social contagions still needs further efforts to discover.

\section{Data Availability}

No data were used to support this study.

\section{Conflicts of Interest}

The authors declare that they have no conflicts of interest.

\section{Acknowledgments}

This work was supported in part by the National Natural Science Foundation of China (Grant nos. 61902359, 61672467, and 61672468), Social Development Project of Zhejiang Provincial Public Technology Research (Grant no. 2016C33168), Zhejiang Provincial Natural Science Foundation of China (Grant no. LQ19F030010), and Opening Project of Shanghai Key Laboratory of Integrated Administration Technologies for Information Security (Grant no. AGK2018001).

\section{References}

[1] P. S. Dodds and D. J. Watts, "Universal behavior in a generalized model of contagion," Physical Review Letters, vol. 92, no. 21, Article ID 218701, 2004.

[2] N. A. Christakis and J. H. Fowler, "The spread of obesity in a large social network over 32 years," New England Journal of Medicine, vol. 357, no. 4, pp. 370-379, 2007.

[3] S. Melnik, J. A. Ward, J. P. Gleeson, and M. A. Porter, "Multistage complex contagions," Chaos: An Interdisciplinary Journal of Nonlinear Science, vol. 23, no. 1, Article ID 013124, 2013.

[4] M. Perc, "Diffusion dynamics and information spreading in multilayer networks: an overview," The European Physical Journal Special Topics, vol. 228, no. 11, pp. 2351-2355, 2019.

[5] G. Cencetti and F. Battiston, "Diffusive behavior of multiplex networks," New Journal of Physics, vol. 21, no. 3, Article ID 035006, 2019.

[6] R. Pastor-Satorras, C. Castellano, P. Van Mieghem, and A. Vespignani, "Epidemic processes in complex networks," Reviews of Modern Physics, vol. 87, no. 3, pp. 925-979, 2015.

[7] J. Borge-Holthoefer and Y. Moreno, "Absence of influential spreaders in rumor dynamics," Physical Review E, vol. 85, Article ID 026116, 2012.
[8] D. Centola, "The spread of behavior in an online social network experiment," Science, vol. 329, no. 5996, pp. 11941197, 2010.

[9] D. Centola, "An experimental study of homophily in the adoption of health behavior," Science, vol. 334, no. 6060, pp. 1269-1272, 2011.

[10] Z.-K. Zhang, C. Liu, X.-X. Zhan, X. Lu, C.-X. Zhang, and Y.-C. Zhang, "Dynamics of information diffusion and its applications on complex networks," Physics Reports, vol. 651, pp. 1-34, 2016.

[11] R. Iyengar, C. Van den Bulte, and J. Y. Lee, "Social contagion in new product trial and repeat," Marketing Science, vol. 34, no. 3, pp. 408-429, 2015.

[12] A. Banerjee, A. G. Chandrasekhar, E. Duflo, and M. O. Jackson, "The diffusion of microfinance," Science, vol. 341, no. 6144, Article ID 1236498, 2013.

[13] X. Guardiola, A. Díaz-Guilera, C. J. Pérez, A. Arenas, and M. Llas, "Modeling diffusion of innovations in a social network," Physical Review E, vol. 66, no. 2, Article ID 026121, 2002.

[14] A. Mellor, M. Mobilia, S. Redner, A. M. Rucklidge, and J. A. Ward, "Influence of luddism on innovation diffusion," Physical Review E, vol. 92, no. 1, Article ID 012806, 2015.

[15] P. Bao, H.-W. Shen, W. Chen, and X.-Q. Cheng, "Cumulative effect in information diffusion: empirical study on a microblogging network," PLoS One, vol. 8, no. 10, Article ID e76027, 2013.

[16] D. J. Watts, "A simple model of global cascades on random networks," Proceedings of the National Academy of Sciences, vol. 99, no. 9, pp. 5766-5771, 2002.

[17] M. S. Granovetter, "The strength of weak ties," American Journal of Sociology, vol. 78, no. 6, pp. 1360-1380, 1973.

[18] W. Wang, M. Tang, H.-F. Zhang, and Y.-C. Lai, "Dynamics of social contagions with memory of non-redundant information," Physical Review E, vol. 92, no. 1, Article ID 012820, 2015.

[19] V.-P. Backlund, J. Saramäki, and R. K. Pan, "Effects of temporal correlations on cascades: threshold models on temporal networks," Physical Review E, vol. 89, no. 6, Article ID 062815, 2014.

[20] M. X. Liu, W. Wang, Y. Liu, M. Tang, S.-M. Cai, and H.-F. Zhang, "Social contagions on time-varying community networks," Physical Review E, vol. 95, no. 5, Article ID 052306, 2017.

[21] S. Unicomb, G. Iñiguez, and M. Karsai, "Threshold driven contagion on weighted networks," Scientific Reports, vol. 8, no. 1, 2018.

[22] W. Wang, M. Tang, P. Shu, and Z. Wang, "Dynamics of social contagions with heterogeneous adoption thresholds: crossover phenomena in phase transition," New Josurnal of Physics, vol. 18, no. 1, Article ID 013029, 2015.

[23] X. Zhu, H. Tian, X. Chen, W. Wang, and S. Cai, "Heterogeneous behavioral adoption in multiplex networks," New Journal of Physics, vol. 20, no. 12, Article ID 125002, 2018.

[24] H. Peng, W. Peng, D. Zhao, W. Wang, Impact of the Heterogeneity of Adoption Thresholds on Behavior Spreading in Complex Networks (on publication), 2020.

[25] W. Wang, H. E. Stanley, and L. A. Braunstein, "Effects of timedelays in the dynamics of social contagions," New Journal of Physics, vol. 20, no. 1, Article ID 013034, 2018.

[26] A. Nematzadeh, E. Ferrara, A. Flammini, and Y.-Y. Ahn, "Optimal network modularity for information diffusion," Physical Review Letters, vol. 113, no. 25, Article ID 088701, 2014.

[27] L. Han, Z. Lin, M. Tang, J. Zhou, Y. Zou, and S. Guan, "Impact of contact preference on social contagions on complex networks," Physical Review E, vol. 101, no. 4, 2020. 
[28] Z. Su, W. Wang, L. Li, H. E. Stanley, and L. A. Braunstein, "Optimal community structure for social contagions," New Journal of Physics, vol. 20, no. 5, Article ID 053053, 2018.

[29] R. Pastor-Satorras and A. Vespignani, "Epidemic dynamics in finite size scale-free networks," Physical Review E, vol. 65, no. 3, Article ID 035108, 2002.

[30] R. Cohen, S. Havlin, and D. ben Avraham, "Efficient immunization strategies for computer networks and populations," Physical Review Letters, vol. 91, no. 24, Article ID 247901, 2003.

[31] N. Madar, T. Kalisky, R. Cohen, D. ben-Avraham, and S. Havlin, "Immunization and epidemic dynamics in complex networks," The European Physical Journal B-Condensed Matter, vol. 38, no. 2, pp. 269-276, 2004.

[32] K. Hu and Y. Tang, "Immunization for scale-free networks by random walker," Chinese Physics, vol. 15, no. 12, pp. 2782-2787, 2006.

[33] L. K. Gallos, F. Liljeros, P. Argyrakis, A. Bunde, and H. Shlomo, "Improving immunization strategies," Physical Review E, vol. 75, no. 4, Article ID 045104, 2007.

[34] Z.-H. Liu, G.-L. Chen, N.-N. Wang, and B. Song, "Greedy immunization strategy in weighted scale-free networks," Engineering Computations, vol. 31, no. 8, pp. 1627-1634, 2014.

[35] C. M. Schneider, T. Mihaljev, S. Havlin, and H. J. Herrmann, "Suppressing epidemics with a limited amount of immunization units," Physical Review E, vol. 84, no. 6, Article ID 061911, 2011.

[36] C. Buono and L. A. Braunstein, "Immunization strategy for epidemic spreading on multilayer networks," EPL (Europhysics Letters), vol. 109, no. 2, p. 26001, 2015.

[37] D. Zhao, L. Wang, S. Li, Z. Wang, L. Wang, and B. Gao, "Immunization of epidemics in multiplex networks," PLoS One, vol. 9, no. 11, Article ID e112018, 2014.

[38] S. Yan, S. Tang, W. Fang, S. Pei, and Z. Zheng, "Global and local targeted immunization in networks with community structure," Journal of Statistical Mechanics: Theory and Experiment, vol. 2015, no. 8, Article ID P08010, 2015.

[39] P. Yuan and S. Tang, "Community-based immunization in opportunistic social networks," Physica A: Statistical Mechanics and Its Applications, vol. 420, pp. 85-97, 2015.

[40] H.-J. Li, C. Zhang, and X.-S. Zhang, "A study of inflammation immunization strategy in weighted complex network," in Proceedings of the 11th International Symposium on Operations Research and its Applications in Engineering, Technology and Management 2013 (ISORA 2013), pp. 1-7, Yellow Mountain, China, August 2013.

[41] B. Lin, W. Guo, and G. Chen, "Edges immunization strategy based on discrete pso in weighted scale-free network," in Proceedings of the 2012 Sixth International Conference on Innovative Mobile and Internet Services in Ubiquitous Computing, pp. 384-389, Palermo, Italy, July 2012.

[42] M. Starnini, A. Machens, C. Cattuto, A. Barrat, and R. PastorSatorras, "Immunization strategies for epidemic processes in time-varying contact networks," Journal of Theoretical Biology, vol. 337, pp. 89-100, 2013.

[43] L. K. Gallos, R. Cohen, P. Argyrakis, A. Bunde, and S. Havlin, "Stability and topology of scale-free networks under attack and defense strategies," Physical Review Letters, vol. 94, no. 18, Article ID 188701, 2005.

[44] X. Yuan, Y. Dai, H. E. Stanley, and S. Havlin, " $k$-core percolation on complex networks: comparing random, localized, and targeted attacks," Physical Review E, vol. 93, no. 6, Article ID 062302, 2016.
[45] X. Huang, J. Gao, S. V. Buldyrev, S. Havlin, and H. E. Stanley, "Robustness of interdependent networks under targeted attack," Physical Review E, vol. 83, no. 6, Article ID 065101, 2011.

[46] J. Shao, S. V. Buldyrev, L. A. Braunstein, S. Havlin, and H. E. Stanley, "Structure of shells in complex networks," Physical Review E, vol. 80, no. 3, Article ID 036105, 2009.

[47] B. Karrer and M. E. J. Newman, "Message passing approach for general epidemic models," Physical Review E, vol. 82, no. 1, Article ID 016101, 2010.

[48] B. Karrer, M. E. J. Newman, and L. Zdeborová, “Percolation on sparse networks," Physical Review Letters, vol. 113, no. 20, Article ID 208702, 2014.

[49] M. Catanzaro, M. Boguñá, and R. Pastor-Satorras, "Generation of uncorrelated random scale-free networks," Physical Review E, vol. 71, no. 2, Article ID 027103, 2005.

[50] M. Schroder, R. D'Souza, D. Sornette, and J. Nagler, "Microtransition cascades to percolation," Physical Review Letters, vol. 112, no. 15, Article ID 155701, 2014. 